\title{
Vigabatrin-associated Reversible MRI Abnormalities in an Infant with Tuberous Sclerosis
}

\author{
Joseph Franklin $\mathrm{Craft}^{1 *}$, Agustin M Cardenas ${ }^{2}$ \\ 1. Department of Radiology, Brookwood Baptist Health Network, Princeton Baptist Medical Center, Birmingham, Alabama, USA \\ 2. Department of Radiology, Children's of Alabama, Birmingham, Alabama, USA \\ * Correspondence: Joseph F. Craft, ATTN: Office of Virginia (Missy) Fadlevich, Grandview Medical Center, 3690 Grandview Pkwy, \\ Birmingham, AL 35243, USA \\ $(-$ joseph.craft@bhsala.com)
}

Radiology Case. 2021 Feb; 15(2):1-6 :: $\quad$ DOI: 10.3941/jrcr.v15i2.3918

\begin{abstract}
Vigabatrin therapy is commonly used in infants diagnosed with tuberous sclerosis complex, particularly in the setting of epilepsy. Utilization of vigabatrin can result in bilateral and symmetric abnormal sequence changes within the deep brain matter and brainstem on magnetic resonance imaging. These abnormalities occur predominantly in infancy, are reversible, and can be asymptomatic or result in symptomatic clinical manifestations. We present a case with classic neuroimaging findings. Familiarity with these findings can prevent unnecessary follow up tests or studies and the cost of continuing or discontinuing vigabatrin therapy should be weighed heavily against the potential manifestation of extrapyramidal symptoms.
\end{abstract}

\section{CASE REPORT}

\section{CASE REPORT}

During a previously unremarkable pregnancy, a 34-weekold male fetus was discovered to have cardiac rhabdomyomas on prenatal ultrasound. After an uneventful full-term delivery, postnatal ultrasound revealed a cyst in the right kidney. A subsequent Magnetic Resonance Image (MRI) of the brain demonstrated multiple subependymal nodules and several scattered cortical tubers, diagnostic of Tuberous Sclerosis Complex (TSC).

The patient displayed unremarkable psychomotor development until the fourth month of life, when he exhibited several documented episodes of right arm extension, head tilting to the right, and short arrest of motor activity lasting about one minute at a time - compatible with complex partial seizures. Due to the refractory nature of these complex partial seizures to conventional antiepileptic treatment, the patient was enrolled in a randomized, double-blind seizure prevention clinical trial for infants with TSC. At the time, vigabatrin (VGB) treatment was initiated with progressive doses reaching $150 \mathrm{mg} / \mathrm{kg} /$ day.

The patient did not experience any additional generalized seizures upon the initiation of VGB until ten months of age, whereupon a routine electroencephalogram (EEG) recorded seizures with breakthrough events, for which the patient was admitted to the hospital for continuous video EEG to determine seizure frequency. A follow-up MRI near discharge revealed a symmetric pattern of diffusion restriction involving the bilateral thalami, subthalamic nuclei, central tegmental tracts and dentate nuclei (Figure 1).

In light of these classic findings, vigabatrin-associated brain abnormalities on MRI were suspected. Despite achieving a low-normal serum value of VGB at the time of discharge of $24.5 \mathrm{ug} / \mathrm{mL}$, (normal 20-160 ug/mL), the decision was made by the clinical team to reduce the patient's dose to 
$100 \mathrm{mg} / \mathrm{kg} /$ day. The patient did not experience any additional seizures over the course of nine months, when a follow-up MRI of the brain showed resolution of the previously seen lesions involving the aforementioned regions (Figure 2).

\section{DISCUSSION}

\section{Vigabatrin}

TSC is characterized by hamartomatous lesions involving multiple organ systems. Some patients with TSC experience infantile spasms or other forms of epilepsy. Vigabatrin is considered first-line therapy in infants with TSC and infantile spasms as well as in pediatric patients aged 10 to 16 years with treatment-refractory complex partial seizures [1,2]. Other patients receive it for compassionate use or Institutional-board approved protocols, as is seen in this case. Other diagnoses that receive VGB therapy include cortical dysplasia, metabolic encephalopathy, and mesial temporal sclerosis [1].

Vigabatrin is a $\gamma$-aminobutyric acid (GABA) analog that irreversibly inhibits $\gamma$-aminobutyric acid transaminase, serving to increase the concentration of GABA in order to depress epileptogenic circuits. VGB is a first-line therapy based on its remarkable effectiveness in reducing seizures, (>95\% reduction), and good tolerability [2]. Initially, there was only one well-known, demonstrated side effect-retinotoxicityresulting in permanent visual field constriction in approximately $30 \%$ of patients after 1 year and termed "vigabatrin-associated visual field loss" (VAVFL) $[3,4]$.

At the time, VAVFL was the limiting factor to the use of VGB, but in more recent years the safety of VGB has been challenged by the emergence and recognition of vigabatrinassociated brain abnormalities on MRI (VABAM) [5].

\section{Etiology \& Demographics:}

VABAM only affects a small subset of the pediatric population. Review of current available literature indicates that vigabatrin associated changes predominantly affect children younger than 2 years, with a mean age of $19.1 \pm 25.6$ months, and affects an estimated $22-32 \%$ of patients with TSC and infantile spasms undergoing treatment with vigabatrin[5]. There is little to no evidence of VABAM affecting individuals over the age of two.

The pathophysiology of VABAM is not well understood. Several animal studies have implicated a demyelination process with associated intramyelinic edema, intracellular cytotoxic edema, and microvacuolation of the deep brain structures [4]. The occurrence of these findings largely in the infancy period has led to the suggestion that brain maturation may play an important role, with vulnerability of immature myelin to the toxicity of vigabatrin a consideration. Other considerations include an indirect infant effect related to elevated GABA levels, or that pathologic neuronal epileptogenic circuitry may make infants susceptible $[5,6]$. Additionally, the distribution of these changes within the deep brain matter may reflect a specific glial or neuronal vulnerability to this medication [4].

\section{Clinical \& Imaging Findings:}

VABAM encompasses a spectrum of changes that represent new-onset vigabatrin-related bilateral and symmetric T2 hyperintensities with varying degrees of diffusion restriction involving any or all of the following structures: the globi pallidi (most commonly affected), thalami, midbrain, dentate nuclei, brainstem, corpus callosum, tectal plate, or medial longitudinal fasciculus $[1,2,3,4]$.

There have been several rare reports in the literature describing neurological symptoms in the setting of VABAM, termed "symptomatic VABAM" [7]. In these cases, the affected areas on MRI studies correlated with patient symptomatology: Extrapyramidal involvement was seen in an infant with dystonia, athetoid movements, and tremor; midbrain/brainstem involvement was seen in an infant with hypotonia and bradycardia; hypervigilance was seen in infant with thalamic involvement. Supplemental associations with headache, drowsiness, fatigue, and dizziness are non-descript, but were also reported. These events are not isolated, and extrapyramidal involvement with related clinical symptomatology has been confirmed in several follow-up studies, including in a retrospective study population of 124 children detailed by Fong et al., whereupon 8 cases of symptomatic VABAM were identified [8]. Fortunately, in all cases VABAM resolved after cessation/reduction of vigabatrin treatment, as was demonstrated in our patient.

Lastly, no statistically significant association of symptomatic VABAM with vigabatrin dosage, (peak or cumulative), was uncovered. However, Hussain et al did suggest a possible association between symptomatic VABAM and concomitant hormonal, (read: "steroidal"), therapy, as is often additionally used in the treatment regimen of epileptic individuals [3]. Thus, assuming symptomatic VABAM is a worse or more severe case of asymptomatic VABAM is too simplified, and one does not necessarily precipitate the other.

\section{Treatment \& Prognosis:}

Little attention has been shifted towards researching vigabatrin-associated brain abnormalities on MRI since their appearance in the literature approximately 15 years ago. In 2017, Hussain et al. set out to identify predictors of these socalled "asymptomatic VBAM," which are seen in approximately $22-32 \%$ of infants taking vigabatrin [3]. Through their retrospective cohort review, tracking 507 brain MRI studies of 257 patients with infantile spasms taking vigabatrin, (10 of whom demonstrated VABAM), Hussain et al. suggested an association that the appearance of asymptomatic VABAM was dose-dependent, linked to peakbut not cumulative-vigabatrin dosage. Meanwhile, an additional retrospective cohort review by Dracopoulos et al. detailing follow-up MRI studies in 25 pediatric patients with asymptomatic VABAM demonstrates the statistically significant, reversible nature of these findings after treatment cessation/reduction [5].

\section{Differential Diagnoses:}

Given that VABAM is a recently identified phenomenon, this abnormal state can easily be confused with other entities that cause deep brain MRI signal changes. This can be 
complicated because there are no necessarily distinguishing neuroimaging findings for several of the following afflictions, including: VABAM, posterior reversible encephalopathy syndrome, West syndrome, or phenytoin toxicity. In fact, several of the aforementioned abnormalities occur in the same patient population as VABAM, and thus will best be differentiated by respective clinical scenarios, i.e. clinical vigabatrin utilization, phenytoin utilization, or clinical history of infantile spasms, pathognomonic EEG pattern, and developmental regression - the triad of findings in West Syndrome. Neuroimaging findings in respect to the above differential are summarized in Table 2 and presented below.

\section{Posterior Reversible Encephalopathy Syndrome}

Common patterns manifest with $\mathrm{T}_{2}$-weighted hyperintensities within the occipital and parietal regions relating to the posterior cerebral artery supply, but nonposterior distribution variants can manifest in watershed areas within the frontal, cerebellar, and brainstem regions.

Other antiepileptic Drug Toxicity (I.e. Phenytoin toxicity) Demyelination of the splenium of the corpus callosum.

\section{West Syndrome}

Transient DWI abnormalities involving the globi pallidi and the dorsal part of the brainstem.

\section{Conclusion}

We present a classic case where $\mathrm{T}_{2}$-hyperintentisies on brain MRI have been associated with the use of vigabatrin in the setting of TSC complicated by a form of epilepsy. VABAM can either manifest as symptomatic or asymptomatic. Familiarity with these neuroimaging findings can prevent unnecessary follow up tests and studies. The cost of precipitating, albeit reversible, extrapyramidal symptoms should be weighed heavily in the practitioner's utilization of vigabatrin therapy.

\section{TEACHING POINT}

Predominantly within infancy, vigabatrin utilization can lead to asymptomatic or symptomatic manifestations of symmetric and bilateral MRI signal hyperintensities with varying degrees of restricted diffusion involving the globi pallidi, brainstem, thalami, anterior commissure, tectum, and dentate nuclei. Asymptomatic VABAM have been demonstrated to be dosedependent and reversible. Symptomatic VABAM is seemingly dose-independent, and potentially associated with concomitant hormonal therapy.

\section{REFERENCES}

1. Sullivan M. MRI is a Must in Children on Vigabatrin Therapy. Pediatric Neurology 2007;10.1016:13.Available at: https://www.mdedge.com/clinicalneurologynews/article/4766 pediatrics/mri-must-children-vigabatrin-therapy. Accessed February 15, 2020.

2. Hsieh D, Thiele E. Vigabatrin-Related Magnetic Resonance Imaging Abnormalities in an Infant with Tuberous Sclerosis Complex and Infantile Spasms. J Pediatr 2013; 162-215. PMID: 22979977.

3. Hussain S, Tsao J, Li M, et al. Risk of Vigabatrinassociated brain abnormalities on MRI in the treatment of infantile spasms is dose-dependent. Epilepsia 2017; 58(4): 674-682. PMID: 28230253.

4. Pearl P, Vezina L, Saneto R, et al. Cerebral MRI abnormalities associated with vigabatrin therapy. Epilepsia 2009; 50(2): 184-194. PMID 18783433.

5. Dracopoulos A, Widjaja E, Raybaud C, Westall CA, Snead OC 3rd. Vigabatrin-associated reversible MRI signal changes in patients with infantile spasms. Epilepsia 2010; 51(7): 1297 1304. PMID: 20384718

6. Schonstedt V, Stecher X, Venegas V, Silva C. Vigabatrininduced MRI changes associated with extrapyramidal symptoms in a child with infantile spasms. Neuroradiol $\mathbf{J}$ 2015; 28(5): 515-518. PMID: 26306928.

7. Dill P, Datta A, Weber P. Are vigabatrin induced T2 hyperintensities in cranial MRI associated with acute encephalopathy and extrapyramidal symptoms? Eur J Paediatr Neurol 2013; 17: 311-315. PMID: 23199677.

8. Fong C, Osborne J, Edwards S, et al. An investigation into the relationship between vigabatrin, movement disorders, and brain magnetic resonance imaging abnormalities in children with infantile spasms. Dev Med Child Neurol 2013; 55: $862-$ 867. PMID: 23789722. 


\section{FIGURES}

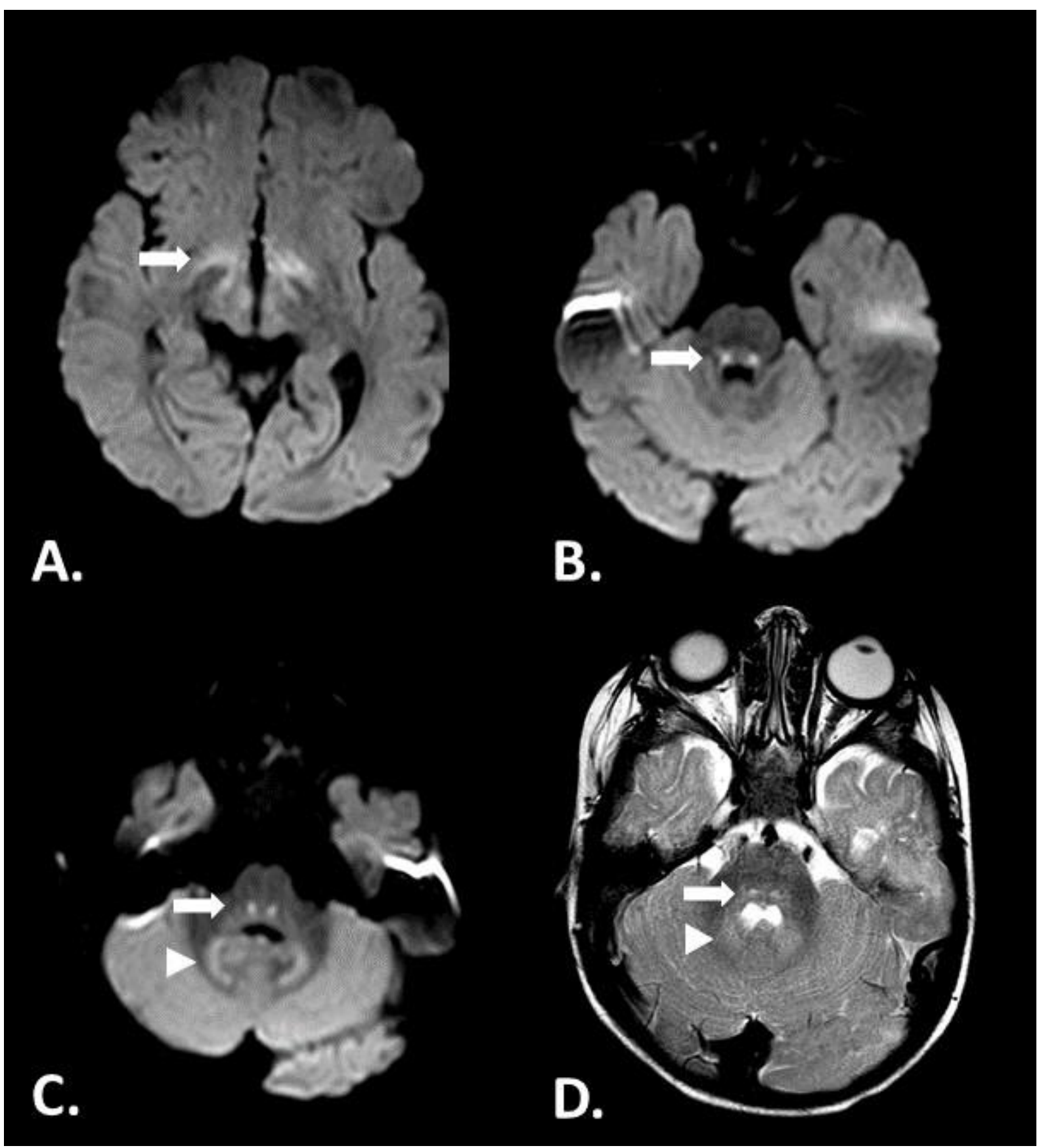

Figure 1: 10-month-old male with tuberous sclerosis and complex partial seizures, treated with vigabatrin at a dosage of $150 \mathrm{mg} / \mathrm{kg} /$ day and subsequent Vigabatrin-associated brain abnormalities.

FINDINGS: Axial Diffusion Weighted Images (1A, 1B, and 1C) and T2 Spin Echo image (1D) depict abnormal T2-weighted signal hyperintensities and restricted diffusion at the level of the bilateral subthalamic nuclei (A, arrow), central tegmental tracts (B,C, and D, arrows), and bilateral dentate nuclei (1C and 1D, arrowheads).

TECHNIQUE: 3 tesla MRI (Philips Intera). Images 1A, 1B, and 1C: axial DWI, b1000, isotropic. Slice thickness: 4mm. Images 1D: axial T2 SE, Tr/TE: 2000/80. Flip angle: 90. Slice thickness: 4mm. 


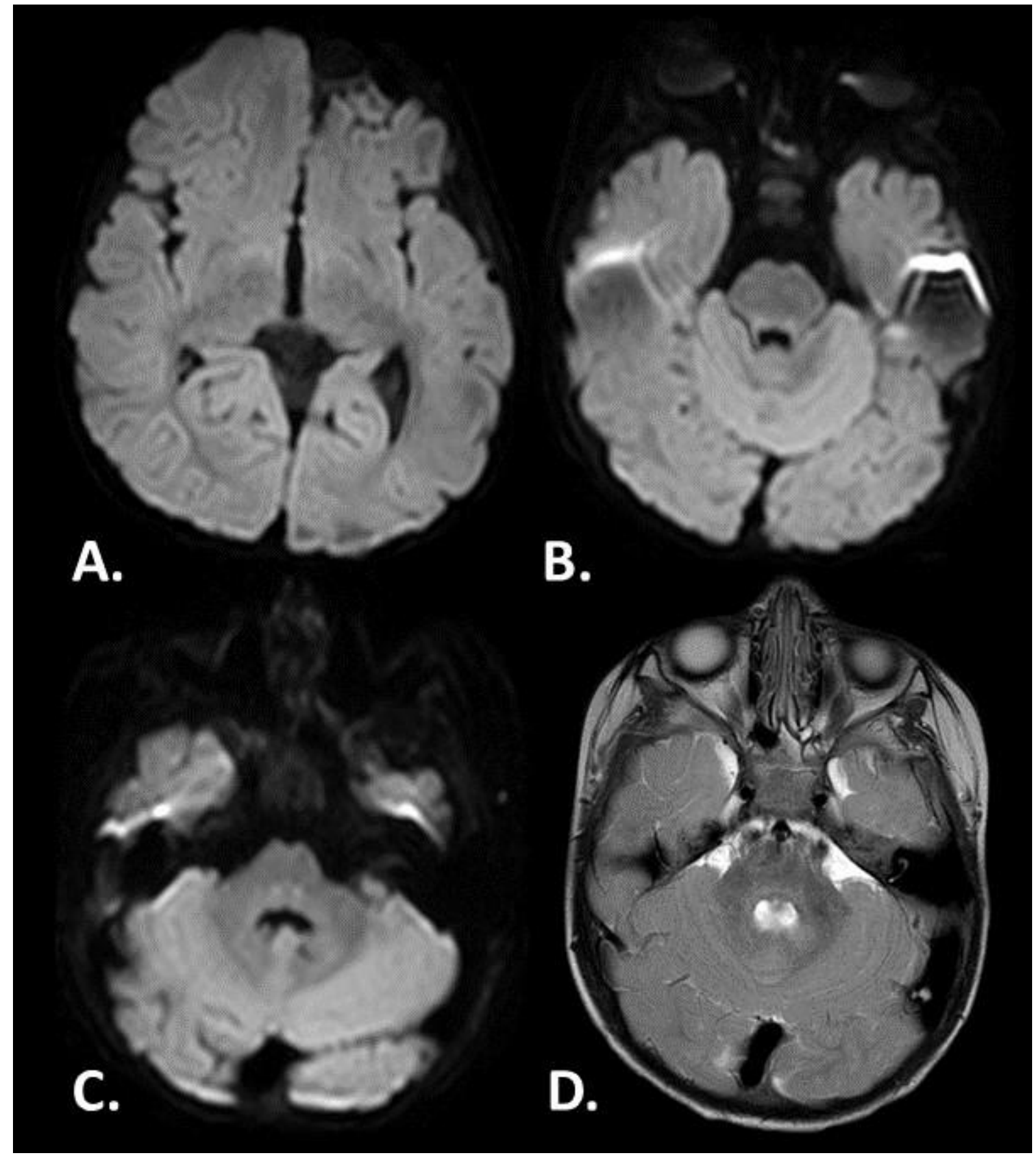

Figure 2: 19-month-old male with tuberous sclerosis and complex partial seizures, treated with vigabatrin at a reduced dosage of $100 \mathrm{mg} / \mathrm{kg} / \mathrm{day}$.

FINDINGS: Axial Diffusion Weighted Images (1A, 1B, and 1C) and T2 Spin Echo image (1D) demonstrate resolution of the abnormal T2-weighted signal hyperintensities and restricted diffusion involving the subthalamic nuclei (A), central tegmental tracts (B,C, and D), and bilateral dentate nuclei (1C and 1D).

TECHNIQUE: 3 tesla MRI (Philips Intera). Images 1A, 1B, and 1C: axial DWI, b1000, isotropic. Slice thickness: 4mm. Images 1D: axial T2 SE, Tr/TE: 2000/80. Flip angle: 90. Slice thickness: 4mm. 


\begin{tabular}{|l|l|}
\hline Etiology & $\begin{array}{l}\text { Undefined etiology; most popular leading theory suggests VGB alters immature myelin formation, } \\
\text { resulting in intramyelinic edema, intracellular cytotoxic edema, and microvacuolation of the deep } \\
\text { brain structures }\end{array}$ \\
\hline Incidence & $22-32 \%$ of patients with infants with TSC and infantile spasms undergoing treatment with VGB. \\
\hline Gender Ratio & No gender predilection \\
\hline Age Predilection & Mean age of $19.1 \pm 25.6$ months \\
\hline Risk Factors & $<2$ years of age, TSC with infantile spasm, undergoing treatment with VGB, high dosage of VGB \\
\hline Treatment & Cessation/reduction of VGB therapy \\
\hline Prognosis & $\begin{array}{l}\text { Quite favorable, although there is limited data as it not usually standard medical practice to obtain } \\
\text { posttreatment MRI. Only a rare set of cases in the literature result in symptomatic VABAM. }\end{array}$ \\
\hline Findings on Imaging & $\begin{array}{l}\text { Bilateral and symmetric T } 2 \text {-weighted signal hyperintensities and restricted diffusion in thalami, } \\
\text { globi pallidi, dentate nuclei, brainstem, tectum, corpus callosum. }\end{array}$ \\
\hline
\end{tabular}

Table 1: Summary table of Vigabatrin-associated MRI abnormalities.

\begin{tabular}{|l|l|}
\hline Diagnosis: & MRI Neuroimaging findings: \\
\hline $\begin{array}{l}\text { VGB-associated MRI brain } \\
\text { abnormalities on MRI (VABAM) }\end{array}$ & $\begin{array}{l}\text { New-onset and reversible bilateral and symmetric } \mathrm{T}_{2} \text {-weighted signal hyperintensities and } \\
\text { restricted diffusion in thalami, globi pallidi, dentate nuclei, brainstem, tectum, corpus } \\
\text { callosum. }\end{array}$ \\
\hline $\begin{array}{l}\text { Posterior Reversible } \\
\text { Encephalopathy Syndrome }\end{array}$ & $\begin{array}{l}\text { Common patterns manifest with } \mathrm{T}_{2} \text {-weighted hyperintensities within the occipital and } \\
\text { parietal regions relating to the posterior cerebral artery supply, but non-posterior } \\
\text { distribution variants can manifest in watershed areas within the frontal, cerebellar, and } \\
\text { brainstem regions. }\end{array}$ \\
\hline $\begin{array}{l}\text { Other antiepileptic Drug Toxicity } \\
\text { (i.e. Phenytoin toxicity) }\end{array}$ & Demyelination of the splenium of the corpus callosum. \\
\hline West Syndrome & $\begin{array}{l}\text { Transient DWI abnormalities involving the globi pallidi and the dorsal part of the } \\
\text { brainstem. }\end{array}$ \\
\hline
\end{tabular}

Table 2: Differential diagnosis table for pathologic processes resulting in similar neuroimaging findings to Vigabatrinassociated MRI abnormalities (VABAM).

\section{ABBREVIATIONS}

$\mathrm{EEG}=$ Electroencephalogram/ Electroencephalography

GABA = Gamma-aminobutyric Acid

MRI = Magnetic Resonance Imaging

TSC $=$ Tuberous Sclerosis Complex

VABAM $=$ VGB-associated brain abnormalities on MRI

VAVFL $=$ VGB-associated visual field loss

VGB $=$ Vigabatrin

\section{KEYWORDS}

Vigabatrin; Vigabatrin Toxicity; VABAM; Vigabatrinassociated brain abnormalities; Tuberous Sclerosis Complex; Pediatric Neuroimaging; MRI

\section{Online access}

This publication is online available at: www.radiologycases.com/index.php/radiologycases/article/view/3918

\section{Peer discussion}

Discuss this manuscript in our protected discussion forum at: www.radiolopolis.com/forums/JRCR

\section{Interactivity}

This publication is available as an interactive article with scroll, window/level, magnify and more features.

Available online at www.RadiologyCases.com

\section{Published by EduRad}

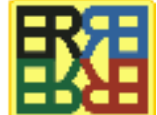

www.EduRad.org 